\title{
Low-Terahertz Transparent Graphene-Based Absorber
}

\author{
Alessandro Giuseppe D'Aloia ${ }^{1,2, *(\mathbb{C})}$, Marcello D'Amore ${ }^{1,2}$ and Maria Sabrina Sarto ${ }^{1,2}$ (D) \\ 1 Department of Astronautical, Electrical and Energy Engineering, Sapienza University of Rome, \\ 00184 Rome, Italy; marcello.damore@uniroma1.it (M.D.); mariasabrina.sarto@uniroma1.it (M.S.S.) \\ 2 Research Center for Nanotechnology applied to Engineering of Sapienza University, 00185 Rome, Italy \\ * Correspondence: alessandrogiuseppe.daloia@uniroma1.it; Tel.: +39-0644-585-806
}

Received: 7 March 2020; Accepted: 17 April 2020; Published: 28 April 2020

\begin{abstract}
A new, transparent, metal-free absorber, based on the use of multilayer graphene/dielectric laminates (GLs), is proposed for applications in the low-terahertz frequency range. The designed absorber has a total thickness of around $70 \mu \mathrm{m}$ and consists of a front matching dielectric layer followed by a GL, a dielectric spacer and a back GL. The laminates are periodic structures constituted of graphene sheets separated by 50-nm-thick polyethylene terephthalate (PET) interlayers, while the matching layer and the spacer are one-quarter-wavelength thick and made of PET. The GLs are modeled as homogeneous-equivalent single layers (ESLs) characterized by their sheet resistances $R_{s}$. An innovative analytical method is proposed in order to select $R_{S}$ values optimizing the electromagnetic wave absorption either in low-gigahertz or low-terahertz frequency range. The frequency spectra of the absorption, reflection and transmission coefficients are computed in the range up to $4 \mathrm{THz}$ by using different values of $R_{s}$. Then, realistic $R_{s}$ values of chemically doped graphene monolayers over PET substrates are considered. The designed absorbers are characterized by an absorption coefficient with a peak value of about 0.8 at the first resonant frequency of $1.1 \mathrm{THz}$, and a $1.4 \mathrm{THz}$ bandwidth centered at $1.5 \mathrm{THz}$ with reflection coefficient below - $10 \mathrm{~dB}$. Moreover, the optical transmittance of the proposed absorbers are computed by means of the optical matrix theory and it is found to be greater than $86 \%$ in all the visible ranges.
\end{abstract}

Keywords: low-terahertz transparent absorber; graphene-polyethylene terephthalate laminate; laminate effective medium model; transmission line approach; absorption; reflection and transmission performance up to $4 \mathrm{THz}$; optical transmittance in the visible range

\section{Introduction}

In the last decade, the exponential growth of wireless communications and data traffic has fueled the research in technologies operating at terahertz (THz) and low- $\mathrm{THz}$ frequency ranges [1]. Moreover, these technologies are attracting ever-growing interest in many other industrial and research fields, such as biomedical imaging and signal detection, as well as stealth materials and sensing [2]. Therefore, intensive efforts have been made to fabricate novel, transparent and multi-functional devices operating at such frequency regimes. In particular, the development of new absorbers operating in the low- $\mathrm{THz}$ and $\mathrm{THz}$ frequency range is generally recognized as an urgent challenge to be addressed, since high absorption is crucial for several applications such as modulators, sensors and solar cells [3,4]. Recently, resonance-based metamaterial absorbers have aroused intensive interest [5,6], since they can offer near unity absorption. However, the complexity of these structures leads to difficulties in fabrication because most of them employ metals, classified as critical raw materials and characterized by electrical and electromagnetic properties hardly tunable, especially when thin films are required [4,7]. Furthermore, most of these structures are not transparent in the visible frequency range, reducing their applications.

In this context, graphene, which is a two-dimensional honeycomb structure of carbon atoms, has gained a lot of interest due to its exceptional electrical, thermal, mechanical and optical properties [8]. 
Currently, two-dimensional graphene layers are characterized by optical transmittance greater than $90 \%$ (as a comparison, the Indium tin oxide transmittance is around $81 \%$ ) and their conductivity can be tuned through electrical or chemical doping.

For instance, single-frequency, dual-frequency and wide-band absorbers made of graphene-based metasurfaces and characterized by large angle tolerance properties in the $\mathrm{THz}$ spectrum up to $4 \mathrm{THz}$ are proposed in [3]. A tunable $\mathrm{THz}$ absorber employing a 5-nm-thick interlayer of $\mathrm{Al}_{2} \mathrm{O}_{3}$ is presented in [9] and an accurate procedure for the design of multilayer $\mathrm{THz}$ absorbers employing tunable graphene metamaterials has been developed in [10], with the aim of obtaining high absorption over a wide- $\mathrm{THz}$ frequency band. Moreover, in [11] tunable double-layer graphene wires are combined with metamaterials having unit cells of cross-shaped metallic resonator in order to realize a polarization-independent absorber with spectral tuning at $\mathrm{THz}$ frequency. Then, a hyperbolic metamaterial consisting of a multilayer structure of alternating graphene and $\mathrm{Al}_{2} \mathrm{O}_{3}$ layers on a $\mathrm{CaF} 2$ substrate is realized and characterized in [12]. More recently, a tunable ultra-broadband $\mathrm{THz}$ absorber based on two layers of graphene ribbons and two different kinds of substrate materials is proposed in [13] while an extensive study on broadband near-perfect absorbers consisting of single-layered and non-structured graphene loaded with periodical arrays of dielectric bricks is presented in [14]. Finally, in [15] a reliable method to extract the electromagnetic constitutive tensors of complex synthesized multilayer graphene-dielectric stacks in the terahertz band is derived.

Nevertheless, the realization of periodic graphene nanostructures is still not an easy task. Recently, Salisbury absorber screens, consisting of a thin resistive film, a dielectric spacer and a backside reflector, have attracted growing interest for the realization of graphene-based $\mathrm{THz}$ absorbers [7,13-18]. In fact, graphene films can act as resistive films [7] and, in addition, their conductivity can be tuned in order to obtain optimum absorption. For instance, numerical studies show that graphene ribbon arrays coupled with a graphene sheet can achieve a near unity absorption in a wide- $\mathrm{THz}$ bandwidth by changing graphene electrical properties through electrical doping due to an applied voltage [14,16]. A similar electrical doping has been investigated in $[4,17]$. Moreover, a new adaptive Salisbury absorbing screen providing a reflection coefficient lower than $-10 \mathrm{~dB}$ in the frequency range $0.6-2.2 \mathrm{THz}$ and with total thickness around $70 \mu \mathrm{m}$ is proposed in [18]. The designed screen, backed by a perfect electrical conducting plate, consists of firstly a graphene layer acting as a lossy sheet and two spacers made of polyethylene terephthalate (PET), separated by an electrically tunable graphene/dielectric laminate (GL). Numerical analyses have been performed on the basis of the equivalent models and the results described in [19-22].

The objective of this paper is the design of new two-periods transparent metal-free absorbers made of multilayer graphene-PET laminates. The materials are chosen in order to obtain an optically transparent screen, and to avoid the use of metals or other critical raw materials, also for the backside reflector, which is usually made of a thin gold layer. The GLs are modelled as homogeneous-equivalent single layers (ESLs) characterized by proper sheet resistances, whose values are obtained analytically in order to achieve good absorption performances. The developed analytical method is innovative since it provides a simple and immediate tool that can be applied to design electromagnetic absorbers in the low-THz frequency range. Finally, the optical transmittances of the backside reflector and of the overall proposed absorbers are computed by means of the well-known matrix theory for the analysis of multilayer systems [23]. It results that the optical transmittance of the designed absorber is greater than $88 \%$ in all the visible ranges.

\section{Absorber Structure}

The schematic structure of the proposed graphene-based absorber is represented in Figure 1. The absorber consists of a front matching PET layer followed by a graphene/PET laminate $L_{1}$, a PET spacer and a back graphene/PET laminate $L_{2}$. The matching layer and the spacer have a one-quarter-wavelength thickness $t_{\mathrm{sp}} ; t_{1}$ and $t_{2}$ are the thicknesses of the laminates $L_{1}$ and $L_{2}$, respectively. 


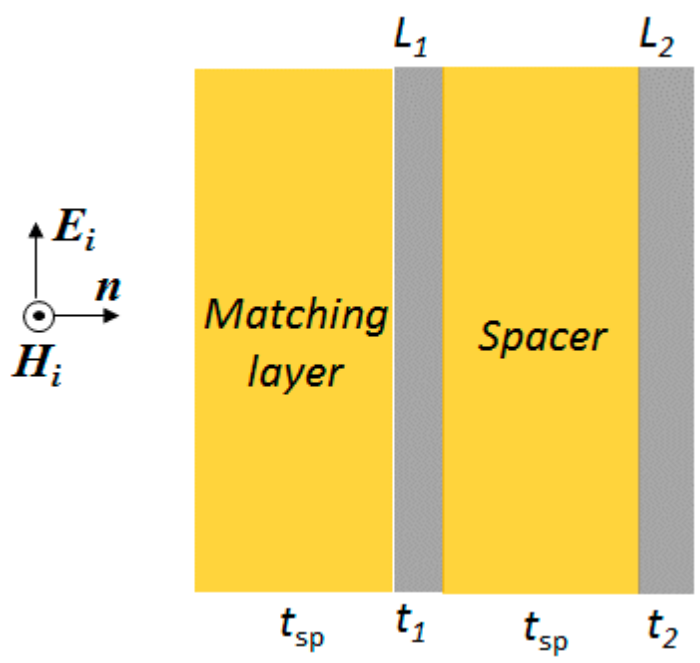

Figure 1. Schematic structure of the cross-section of the planar absorber illuminated by a plane wave.

The absorber is assumed to have infinite transverse dimensions, and to be illuminated by a plane wave with normal incidence.

PET is selected for the realization of the spacer and matching layer due to its mechanical properties, optical transparency and cost-effectiveness. Moreover, graphene films over PET substrates are nowadays commercially available and they are characterized by outstanding electrical and mechanical properties [24]. Another polymer commonly used as substrate for graphene is polymethylmethacrylate (PMMA). However, PMMA films are characterized by worse mechanical properties than PET ones.

The $i$ th laminate $L_{i}$ of Figure 2a consists of a periodic structure constituted of $N_{i}$ bilayers made of a graphene sheet separated by a PET interlayer having thickness $t_{i n t}$. Thus, the thickness of each graphene-PET bilayer is $t_{\mathrm{b}}=\delta+t_{i n t}, \delta$ being the graphene thickness, conventionally assumed equal to $0.34 \mathrm{~nm}$. It results $t_{\mathrm{b}} \cong t_{\text {int }}$ and the total thickness of the $i$ th laminate, $t_{i}$, is equal to $N_{i} t_{\text {int }}$.
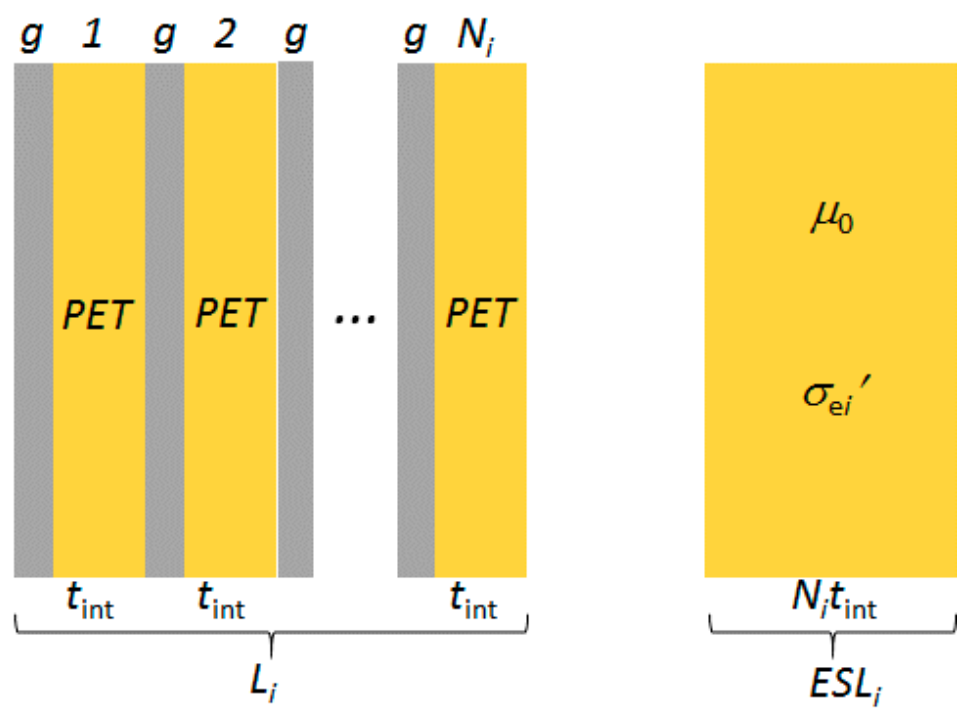

(a)

(b)

Figure 2. Schematic structure of (a) graphene-polyethylene terephthalate (PET) laminate $L_{i}$ and (b) its equivalent single layer $\left(\mathrm{ESL}_{i}\right)$. 


\section{Graphene and Polymer Modeling}

\subsection{Graphene Conductivity}

The frequency-dependent conductivity $\sigma(\omega)$ of an isolated graphene sheet can be represented by Kubo's formalism, which takes into account the intraband and the interband contributions [25].

In the low-THz frequency band only the intraband conductivity term is considered, since the interband one is negligible. In fact it results $\hbar \omega<<2\left|\mu_{c}\right|$, i.e., $f<<f_{c} \approx 0.484 \times 10^{15}\left|\mu_{c}\right|$, where $\mu_{c}$ is the chemical potential in $\mathrm{eV}, \hbar=h / 2 \pi$ the reduced Planck's constant (with $h=6.6262 \times 10^{-34} \mathrm{Js}$ ) and $\omega$ the angular frequency. It yields [25]:

$$
\sigma(\omega)=-j \frac{e^{2} k_{B} T}{\pi \hbar^{2}(\omega-j / \tau)}\left[\frac{\mu_{c}}{k_{B} T}+2 \ln \left(e^{-\mu_{c} /\left(k_{B} T\right)}+1\right)\right]
$$

in which $e=1.602 \times 10^{-19} \mathrm{C}$ is the elementary electron charge, $k_{B}=1.38064 \times 10^{-23} \mathrm{~J} / \mathrm{K}$ is the Boltzmann's constant, $T$ is the temperature in Kelvin and $\tau$ is the relaxation time of graphene.

It is worth noticing that if $\left|\mu_{c}\right|>>k_{B} T$, i.e., if $\left|\mu_{c}\right|>>0.0259 \mathrm{eV}$ at $T=300 \mathrm{~K}$, the exponential term in (1) becomes negligible and the intraband conductivity can be approximated by the following semi-classical Drude model:

$$
\sigma(\omega)=\frac{\sigma}{1+j \omega \tau}
$$

where $\sigma$ is the dc conductivity:

$$
\sigma=\frac{e^{2} \mu_{c} \tau}{\pi \hbar^{2}}
$$

The scattering time can be expressed as a function of $\mu_{c}$ and of the carrier mobility $\mu$ in the following form [22]:

$$
\tau=\frac{\mu \mu_{c}}{e v_{F}^{2}}
$$

in which $v_{F}=10^{6} \mathrm{~m} / \mathrm{s}$ is the Fermi velocity. Therefore, Equation (3) can be rewritten as:

$$
\sigma=\frac{e \mu \mu_{c}^{2}}{\pi \hbar^{2} v_{F}^{2}}
$$

The graphene dc conductivity $\sigma$ at zero-field and zero-charge density is estimated to be close to the conductivity quantum $0.38 \times 10^{-4} \mathrm{~S}$ and can reach the value of a few $\mathrm{mS}$ by increasing the chemical potential $\mu_{c}$ through chemical or electrical doping [24].

The graphene layers composing the GL are assumed to be decoupled electronically thanks to the presence of dielectric interlayers with a thickness in the order of some tens of nanometers. Moreover, due to the infinite planar dimensions of the homogeneous graphene monolayers, plasmonic surface waves are neglected $[26,27]$.

\subsection{Polymer Permittivity}

The PET-frequency-dependent relative permittivity can be predicted by using the Havriliak-Negami model [28]:

$$
\varepsilon_{\mathrm{r}}(\omega)=\varepsilon_{\mathrm{r} \infty}+\frac{\Delta \varepsilon_{\mathrm{R}}}{\left[1+\left(j \omega \tau_{\mathrm{R}}\right)^{\beta}\right]^{\alpha}}
$$

where $\varepsilon_{\mathrm{r} \infty}$ is the high-frequency value, $\Delta \varepsilon_{\mathrm{R}}$ the relaxation strength, $\tau_{\mathrm{R}}$ the relaxation time and $\alpha$ and $\beta$ fitting parameters. According to [29], the best fit with experimental data is obtained when $\varepsilon_{\mathrm{r} \infty}=2.54$, $\Delta \varepsilon_{\mathrm{R}}=0.46, \tau_{\mathrm{R}}=0.05 \mathrm{ps}, \alpha=2.9$ and $\beta=0.9$. 
Thus, in the low-THz frequency range, the PET permittivity $\varepsilon_{\mathrm{r}}(\omega)$ is a complex number, with a real part $\varepsilon_{\mathrm{r} R}(\omega)$ and an imaginary part $\varepsilon_{\mathrm{rI}}(\omega)$, that are frequency-dependent quantities. It yields:

$$
\varepsilon_{\mathrm{r}}(\omega)=\varepsilon_{\mathrm{rR}}(\omega)+j \varepsilon_{\mathrm{rI}}(\omega)
$$

The frequency spectra of $\varepsilon_{\mathrm{r} R}(\omega)$ and $\varepsilon_{\mathrm{rI}}(\omega)$ are shown in Figure $3 \mathrm{a}, \mathrm{b}$ in the range from $0.01 \mathrm{THz}$ up to $4 \mathrm{THz}$.

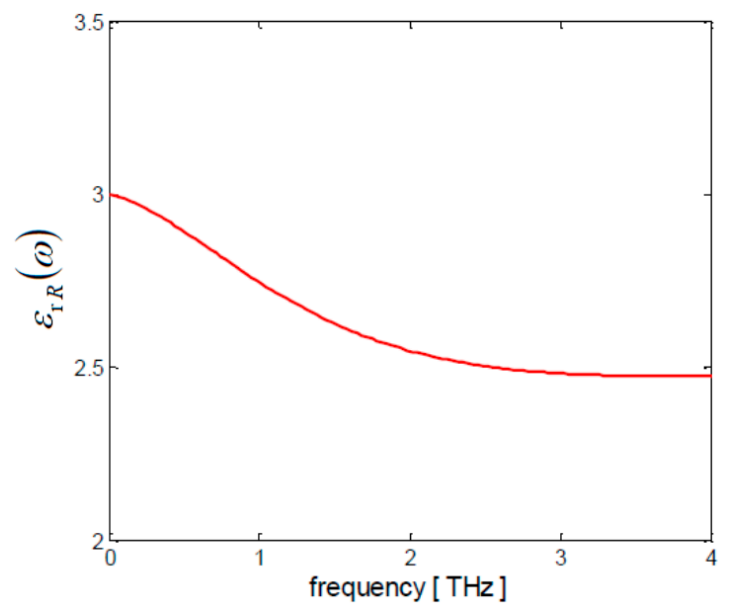

(a)

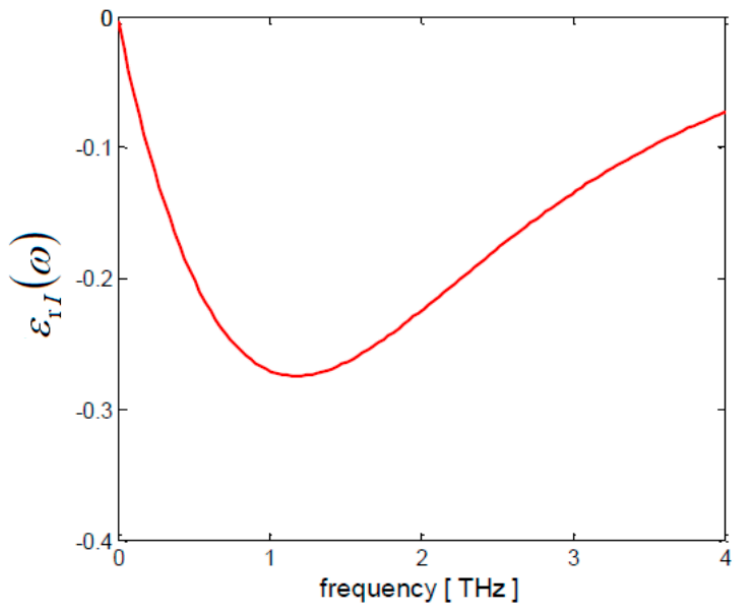

(b)

Figure 3. Frequency spectra of the (a) real and (b) imaginary part of the relative permittivity of PET polymer substrate.

\subsection{Optical Transmittance of Graphene and PET Laminate}

According to [30,31], the optical transmittance $T_{\text {opt }} \lambda=550 \mathrm{~nm}$ at the light wavelength $\lambda$ equal to $550 \mathrm{~nm}$ of stacked graphene monolayers is given by:

$$
T_{\text {opt } \lambda=550 \mathrm{~nm}}=(1+1.13 N \pi \gamma / 2)^{-2}
$$

where $N$ is number of suspended graphene layers and $\gamma$ is the fine structure constant, equal to $1 / 137$ [32]. Thus, for a single layer the optical transmittance is $97 \%$, whereas for $N=4$ or $N=5$ it is $90 \%$ or $88 \%$. However, such transmittance values do not consider the substrate effects that may not be neglected in case of graphene-based structures, like the ones sketched in Figures 1 and 2a.

Indeed, the optical transmittance of GLs can be computed through the standard thin-film optics approach, based on the Fresnel equations [23]. For such calculations, a complex refractive index $\widetilde{n}_{g}$ is typically assumed for graphene [33], with real part $n_{g}$ corresponding to the graphene refractive index and imaginary part $k_{g}$ representing the graphene extinction coefficient. It yields:

$$
\tilde{n}_{g}=n_{g}-j k_{g}
$$

Very recent experiments have shown that in the visible range, $n_{g}$ is constant and equal to 3 , while the graphene extinction coefficient is given by:

$$
k_{g}=\frac{\zeta}{3} \mathrm{C}
$$

with $\zeta$ being a constant equal to $5.446 \mu \mathrm{m}^{-1}$ and $\lambda$ the incident light wavelength expressed in micrometers [34]. 
Concerning the dielectric layers, for a typical PET sample the complex refractive index $\widetilde{n}_{P E T}$ is equal to the refractive index $n_{P E T}$ (a real number), since the PET extinction coefficient $k_{P E T}$ is zero in the visible range [35].

The graphene and PET refractive indices and extinction coefficients are reported in Figure $4 a, b$, respectively, for wavelengths ranging between $400 \mathrm{~nm}$ and $700 \mathrm{~nm}$.

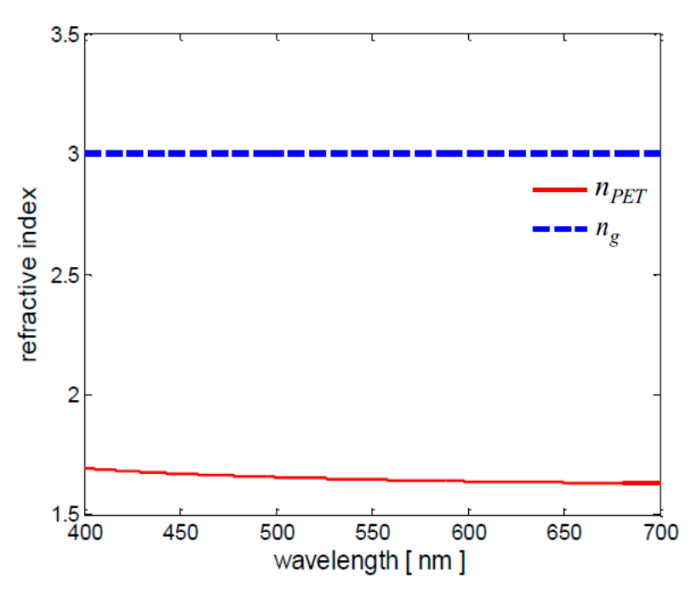

(a)

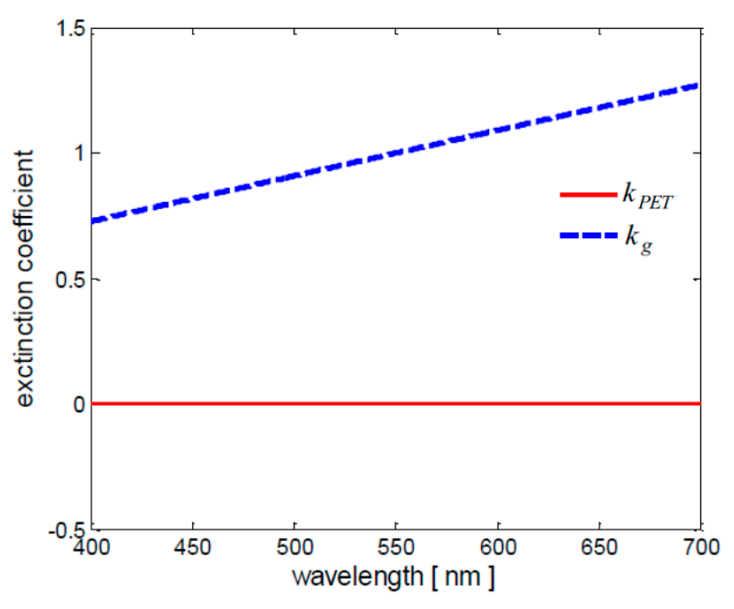

(b)

Figure 4. Refractive index (a) and extinction coefficient (b) of graphene (- - ) and PET (-) in the visible range.

\section{Transmission Wave Modeling}

\subsection{Multilayer Model}

The overall transfer matrix [ $\Phi]$ of the absorber structure sketched in Figure 1 is [19]:

$$
[\Phi]=\left[\Phi_{\mathrm{sp}}\right]\left[\Phi_{L 1}\right]\left[\Phi_{\mathrm{sp}}\right]\left[\Phi_{L 2}\right]
$$

in which $\left[\Phi_{L i}\right]$ and $\left[\Phi_{\mathrm{sp}}\right]$ are the transfer matrices of the ith laminate $L_{i}$ and of the PET layers (either the spacer or the front matching layer).

The transfer matrix $\left[\Phi_{L i}\right]$ is given by:

$$
\left[\Phi_{L i}\right]=\prod^{N_{i}}\left[\Phi_{\mathrm{g} i}\right]\left[\Phi_{\mathrm{int}}\right]
$$

where $\left[\Phi_{g i}\right]$ is the short-line transfer matrix of the $i$ th single graphene layer with conductivity $\sigma_{i}(\omega)$ :

$$
\left[\Phi_{\mathrm{g} i}\right]=\left[\begin{array}{cc}
1 & 0 \\
\sigma_{i}(\omega) & 1
\end{array}\right]
$$

and $\left[\Phi_{\text {int }}\right]$ is the transfer matrix of the PET dielectric interlayer:

$$
\left[\Phi_{\text {int }}\right]=\left[\begin{array}{cc}
\cos \alpha_{\text {int }} & j \eta_{s} \sin \alpha_{\text {int }} \\
j \sin \alpha_{\text {int }} / \eta_{s} & \cos \alpha_{\text {int }}
\end{array}\right]
$$

The attenuation constant $\alpha_{\text {int }}$, the wavelength $\lambda_{S}$ and the characteristic impedance $\eta_{S}$ of the PET layers are respectively:

$$
\alpha_{\text {int }}=2 \pi t_{\text {int }} / \lambda_{S}
$$




$$
\begin{aligned}
& \lambda_{S}=\frac{c_{0} \sqrt{2}}{f \sqrt{\varepsilon_{\mathrm{r} R}(\omega)+\left|\varepsilon_{\mathrm{r}}(\omega)\right|}} \\
& \eta_{S}=\frac{\eta_{0}}{f \sqrt{\varepsilon_{\mathrm{r} R}(\omega)+j \varepsilon_{\mathrm{rI}}(\omega)}}
\end{aligned}
$$

The transfer matrix $\left[\Phi_{\mathrm{sp}}\right]$ of the PET spacer and matching layer is given by Equation (14) replacing $\alpha_{\text {int }}$ with $\alpha_{\mathrm{sp}}=2 \pi t_{\mathrm{sp}} / \lambda_{S}$.

\subsection{Equivalent Single-Layer Model of the Graphene-PET Laminate}

In the low-THz frequency range, the PET interlayers with $t_{\text {int }}=50 \mathrm{~nm}$ behave as short lines (SLs), since it results $\left(t_{\mathrm{int}} / \lambda_{s}\right)<<1$. Thus, the condition required to apply the homogenization procedure to the periodic structure given by the graphene-PET laminate of Figure 2a is satisfied [36]. Consequently, each multilayer laminate $L_{i}$ can be represented by the corresponding ESL $\mathrm{E}_{i}$ having thickness $t_{i}=N_{i} t_{\text {int }}$, as shown in Figure $2 \mathrm{~b}$. The homogeneous $\mathrm{ESL}_{i}$ is characterized by permeability $\mu_{0}$ and effective conductivity $\sigma_{e i}^{\prime}(\omega)$ expressed in s/m [36], given by:

$$
\sigma_{\mathrm{e} i}^{\prime}(\omega)=\frac{\sigma_{i}(\omega)}{t_{\mathrm{int}}}+j \omega \varepsilon_{0} \varepsilon_{\mathrm{r}}(\omega)
$$

that can be rewritten as:

$$
\sigma^{\prime}{ }_{e i}(\omega)=\sigma^{\prime}{ }_{e i R}(\omega)+j \sigma^{\prime}{ }_{e i I}(\omega)
$$

where the real and imaginary components, respectively $\sigma^{\prime}{ }_{\text {eiR }}(\omega)$ and $\sigma^{\prime}$ eiI $(\omega)$, are:

$$
\begin{gathered}
\sigma^{\prime}{ }_{\mathrm{e} i R}(\omega)=\frac{\sigma_{i}}{t_{\mathrm{int}}\left[1+(\omega \tau)^{2}\right]}-\omega \varepsilon_{0} \varepsilon_{\mathrm{rI}}(\omega) \\
\sigma^{\prime}{ }_{\mathrm{e} i I}(\omega)=\omega\left[\varepsilon_{0} \varepsilon_{\mathrm{r} R}(\omega)-\frac{\sigma_{i} \tau}{t_{\mathrm{int}}\left[1+(\omega \tau)^{2}\right]}\right]
\end{gathered}
$$

The transfer matrix $\left[\Phi_{\mathrm{ESL} i}\right]$ of the homogeneous $\mathrm{ESL}_{i}$ is:

$$
\left[\Phi_{\mathrm{ESL} i}\right]=\left[\begin{array}{cc}
\cosh \left(m_{i} t_{i}\right) & \eta_{i} \sinh \left(m_{i} t_{i}\right) \\
\sinh \left(m_{i} t_{i}\right) / \eta_{i} & \cosh \left(m_{i} t_{i}\right)
\end{array}\right]
$$

where $m_{i}$ and $\eta_{i}$ are the ESL $i$ propagation constant and wave impedance, respectively given by:

$$
\begin{aligned}
& m_{i}=\sqrt{j \omega \mu_{0} \sigma^{\prime}{ }^{\prime} i(\omega)} \\
& \eta_{i}=\sqrt{j \omega \mu_{0} / \sigma^{\prime}{ }_{\mathrm{e} i}(\omega)}
\end{aligned}
$$

Note that the $\mathrm{ESL}_{i}$ behaves as an SL since $\left(t_{i} / \lambda_{\mathrm{ESL}} i\right)<<0.1$ in the considered frequency range. The wavelength $\lambda_{\mathrm{ESL} i}$ assumes the following new expression:

$$
\lambda_{\mathrm{ESL} i}=2 \pi\left\{\sqrt{\frac{\omega \mu_{0}}{2}\left[\sigma^{\prime}{ }_{\mathrm{e} i I}(\omega)+\left|\sigma^{\prime}{ }_{\mathrm{e} i}(\omega)\right|\right]}\right\}^{-1}
$$

Thus, in the case of SL hypothesis, the $\mathrm{ESL}_{i}$ transfer matrix expressed in Equation (22) becomes:

$$
\left[\Phi_{\mathrm{ESL} i}\right]_{\mathrm{SL}}=\left[\begin{array}{cc}
1 & j \omega \mu_{0} t_{i} \\
\sigma_{\mathrm{e} i}(\omega) & 1
\end{array}\right]
$$


where $\sigma_{e i}(\omega)$ is the equivalent effective conductivity, expressed in Siemens and computed as:

$$
\sigma_{\mathrm{e} i}(\omega)=N_{i} t_{\mathrm{int}} \sigma^{\prime}{ }_{\mathrm{e} i}
$$

Considering Equation (18), the equivalent effective conductivity can be rewritten as:

$$
\sigma_{\mathrm{ei}}(\omega)=\frac{N_{i} \sigma_{i}}{1+j \omega \tau}+j \omega N_{i} t_{\mathrm{int}} \varepsilon_{0} \varepsilon_{\mathrm{r}}(\omega)
$$

\subsection{Reflection, Transmission and Absorption Coefficients}

The reflection $R$ and transmission $T$ coefficients of a multilayer absorber are given by the following expressions [18]:

$$
\begin{gathered}
R=\left|\frac{1-Y_{\text {in }} \eta_{0}}{1+Y_{\text {in }} \eta_{0}}\right| \\
T=2\left|\Phi_{11}+\Phi_{22}+\eta_{0} \Phi_{21}+\Phi_{12} / \eta_{0}\right|^{-1}
\end{gathered}
$$

in which $Y_{i n}$ is the absorber input admittance:

$$
Y_{i n}=\frac{\eta_{0} \Phi_{21}+\Phi_{22}}{\eta_{0} \Phi_{11}+\Phi_{12}}
$$

and $\Phi_{11}, \Phi_{22}, \Phi_{12}, \Phi_{21}$ are the coefficients of the absorber overall transfer matrix (11). If the SL hypothesis $\left(t_{i} / \lambda_{\mathrm{ESL} i}\right)<<0.1$ is satisfied, the overall transfer matrix $[\Phi]$ can be computed using matrices $\left[\Phi_{\mathrm{ESL} i}\right]_{\mathrm{SL}}$ instead of matrices $\left[\Phi_{\mathrm{L} i}\right]$ without losing accuracy.

Finally, the reflection coefficient in decibel $R_{d B}$ and the absorption coefficient $A$ are obtained from $R$ and $T$ as:

$$
\begin{gathered}
R_{d B}=20 \log (R) \\
A=1-T-R
\end{gathered}
$$

\section{Sheet Resistances of Graphene-PET Laminates}

The absorption performances of the proposed transparent screens depend strongly on the laminate-sheet resistances that are functions of the number $N_{i}$ and dc conductivity $\sigma_{i}$ of the graphene layers. In order to obtain the optimum values of the sheet resistances, a new analytical formulation is developed on the basis of previously obtained results [37].

Each laminate $L_{i}$ is modeled as the corresponding homogeneous $\mathrm{ESL}_{i}$ represented by the $\mathrm{SL}$ transfer matrix [ $\left.\Phi_{\mathrm{ESL} i}\right]_{\mathrm{SL}}$ expressed in Equation (26), in which the off-diagonal coefficients are $j \omega \mu_{0} t_{i}$ and $\sigma_{\mathrm{e} i}(\omega)$. Assuming the low-frequency hypothesis, the term $j \omega \mu_{0} t_{i}$ can be neglected, being the total thickness of the $\mathrm{ESL}_{i}$ in nanometric scale. Moreover, the equivalent effective conductivity $\sigma_{e i}(\omega)$ in Equation (28) can be approximated with the term $N_{i} \sigma_{i}$. Therefore, the transfer matrix [ $\left.\Phi_{\mathrm{ESL} i}\right]_{\mathrm{SL}}$ assumes the following simplified form.

$$
\left[\Phi_{\mathrm{ESL} i}\right]_{\mathrm{SL}}=\left[\begin{array}{cc}
1 & 0 \\
G_{s i} & 1
\end{array}\right]
$$

where the sheet conductance $G_{s i}$ can be written as:

$$
G_{s i}=N_{i} \sigma_{i}
$$

The thickness of the spacer and matching layer sketched in Figure 1 is assumed equal to $t_{\mathrm{sp}}=\lambda_{\mathrm{sr}} / 4$ in which $\lambda_{s r}=c_{0} / f_{r} \sqrt{\varepsilon_{\mathrm{r}}}$ is the wavelength at the resonant frequency $f_{\mathrm{r}}$, being $\varepsilon_{\mathrm{r}}=\varepsilon_{\mathrm{r} \infty}+\Delta \varepsilon_{\mathrm{R}}$, 
the low-frequency PET permittivity. Therefore, the transfer matrix $\left[\Phi_{\mathrm{sp}}\right]_{\mathrm{r}}$ at the resonance frequency assumes the following form:

$$
\left[\Phi_{\mathrm{sp}}\right]_{\mathrm{r}}=\left[\begin{array}{cc}
0 & j \eta_{s} \\
j / \eta_{s} & 0
\end{array}\right]
$$

in which $\eta_{s}=\eta_{0} / \sqrt{\varepsilon_{\mathrm{r}}}$. Thus, the overall transfer matrix at the resonant frequency $[\Phi]_{\mathrm{r}}$ is computed as:

$$
[\Phi]_{\mathrm{r}}=\left[\begin{array}{cc}
0 & j \eta_{s} \\
j / \eta_{s} & 0
\end{array}\right]\left[\begin{array}{cc}
1 & 0 \\
G_{s 1} & 1
\end{array}\right]\left[\begin{array}{cc}
0 & j \eta_{s} \\
j / \eta_{s} & 0
\end{array}\right]\left[\begin{array}{cc}
1 & 0 \\
G_{s 2} & 1
\end{array}\right]
$$

The coefficients of matrix (37) are used to evaluate the corresponding input admittance $Y_{i n r}$ and the transmission coefficient $T_{\mathrm{r}}$. These quantities are expressed as functions of the sheet conductances $G_{s 1}$ and $G_{s 2}$. It yields:

$$
\begin{gathered}
Y_{i n \mathrm{r}}=\left[G_{s 2}+1 / \eta_{0}\right] \times\left[1+G_{s 1} G_{s 2} \eta_{0}^{2} / \varepsilon_{\mathrm{r}}+G_{s 1} \eta_{0} / \varepsilon_{\mathrm{r}}\right]^{-1} \\
T_{\mathrm{r}}=2\left[2+\eta_{0} G_{s 2}+\left(G_{s 1} \eta_{0}^{2} / \varepsilon_{\mathrm{r}}\right)\left(G_{s 2}+1 / \eta_{0}\right)\right]^{-1}
\end{gathered}
$$

Setting $Y_{\text {inr }}$ and $T_{\mathrm{r}}$ respectively equal to $1 / \eta_{0}$ and $T^{*}$, where $T^{*}$ is a target value of the transmission coefficient at frequency $f_{\mathrm{r}}$, it is possible to obtain the optimum laminate-sheet conductances. It yields:

$$
\begin{aligned}
& G_{s 1}=\frac{\varepsilon_{\mathrm{r}}}{\eta_{0}}\left(\frac{1-2 T^{*}}{1-T^{*}}\right) \\
& G_{s 2}=\frac{1}{\eta_{0}}\left(\frac{1-2 T^{*}}{T^{*}}\right)
\end{aligned}
$$

with $T^{*}<0.5$. Notice that the latter equations can be used also in the low-gigahertz frequency range, as discussed in [37].

Considering a perfect electric conductor (PEC) back plate (i.e., $G_{s 2} \rightarrow \infty$ ) instead of a graphene-dielectric laminate, Equation (38) and Equation (39) become respectively:

$$
\begin{gathered}
Y_{i n \mathrm{r}}=\varepsilon_{\mathrm{r}} /\left(\eta_{0}^{2} G_{s 1}\right) \\
T_{\mathrm{r}}=0
\end{gathered}
$$

Moreover, setting $Y_{\text {inr }}$ equal to $1 / \eta_{0}$, the sheet conductance of the first laminate is obtained from Equation (42) as:

$$
G_{s 1}=\varepsilon_{\mathrm{r}} / \eta_{0}
$$

With the spacer thickness $t_{\mathrm{sp}}$ being equal to $\lambda_{s} / 4$, the peak values of the absorption coefficient occur at the following resonant frequency:

$$
f_{\mathrm{r}}=\frac{c_{0}}{4 t_{\mathrm{sp}} \sqrt{\varepsilon_{\mathrm{r}}}}
$$

It should be noted that the low-frequency graphene conductivity and PET permittivity considered in the previous equations decrease in the low- $\mathrm{THz}$ frequency band. Thus, the values of sheet conductances given by Equations (40), (41) or (44) cannot be considered as the ones that optimize the absorption performances, but as initial reference values in the design process.

\section{Optical Transmittance Modeling}

The optical transmittance $T_{\text {opt }}$ of a material is defined as the ratio between the light intensity transmitted by the material to that incident upon it. The most general approach to compute $T_{\text {opt }}$ is based on a matrix formulation of the boundary conditions at the layer surfaces [23]. 
In case of a normally incident beam of light on a multilayer structure placed in air, the optical transmittance $T_{\text {opt }}$ can be computed as [38]:

$$
T_{\mathrm{opt}}=\left|\frac{2 n_{0}}{n_{0} B+C}\right|^{2}
$$

where $n_{0}$ is the air effective refractive index, conventionally equal to 1 , and $B$ and $C$ are the normalized total tangential electric and magnetic fields at the front interface of the absorbing structure sketched in Figure 1 [23]. According to the optical matrix formulation, $B$ and $C$ are given by:

$$
\left[\begin{array}{l}
B \\
C
\end{array}\right]=[M]\left[\begin{array}{c}
1 \\
n_{0}
\end{array}\right]
$$

with $[M]$ being the overall ray transfer matrix of the considered structure [23].

The ray transfer matrix $\left[M_{L i}\right]$ of the graphene laminate sketched in Figure 2a consisting of $N_{i}$ graphene sheets separated by $N_{i}$ PET interlayers is computed as:

$$
\left[M_{L i}\right]=\prod^{N_{i}}\left(\left[M_{g}\right]\left[M_{\mathrm{int}}\right]\right)
$$

where $\left[M_{g}\right]$ and $\left[M_{\text {int }}\right]$ are the ray transfer matrices of the graphene layer and of the PET interlayer. In particular, $\left[M_{g}\right]$ is

$$
\left[M_{g}\right]=\left[\begin{array}{cc}
\cos \delta_{g} & j \sin \delta_{g} / \widetilde{n}_{g} \\
j \sin \delta_{g} \widetilde{n}_{g} & \cos \delta_{g}
\end{array}\right]
$$

With

$$
\delta_{g}=\frac{2 \pi}{\lambda}\left(\widetilde{n}_{g} t_{g}\right)
$$

being $t_{g}$ and $\left.\widetilde{n}_{g} t_{g}\right)$ the conventional and the effective optical thickness of a graphene layer. Then, matrix $\left[M_{\text {int }}\right]$ is obtained from Equations (49) and (50) replacing the graphene complex refractive index $\tilde{n}_{g}$ and thickness $t_{\mathrm{g}}$ with the PET complex refractive index $\widetilde{n}_{P E T}$ and thickness $t_{\text {int }}$.

Finally, the overall ray transfer matrix $[M]$ of the absorber structure sketched in Figure 1, is:

$$
[M]=\left[M_{\mathrm{sp}}\right]\left[M_{L 1}\right]\left[M_{\mathrm{sp}}\right]\left[M_{L 2}\right]
$$

where matrix $\left[M_{\mathrm{sp}}\right]$ of the PET spacer and matching layer is obtained from matrix $\left[M_{\mathrm{int}}\right]$ replacing $t_{\text {int }}$ with $t_{\mathrm{sp}}$.

\section{Absorber Performance}

The sheet conductances of the laminates $L_{1}$ and $L_{2}$ are computed by means of Equations (40) and (41) assuming $\varepsilon_{\mathrm{r}}=3$ and $T^{*}$ equal to $0.1,0.07$ and 0.05 . The obtained values of the corresponding sheet resistances $R_{s i}=1 / G_{s i}$ are reported in Table 1 .

Table 1. Sheet Resistances of Graphene-PET Laminates.

\begin{tabular}{ccc}
\hline $\boldsymbol{T}^{*}$ & $\begin{array}{c}\boldsymbol{R}_{\boldsymbol{s} \mathbf{1}} \\
{[\boldsymbol{\Omega} / \mathbf{s q}]}\end{array}$ & $\begin{array}{c}\boldsymbol{R}_{\boldsymbol{s} \mathbf{2}} \\
{[\boldsymbol{\Omega} / \mathbf{s q}]}\end{array}$ \\
\hline 0.10 & 141 & 47 \\
0.07 & 135 & 31 \\
0.05 & 133 & 21 \\
\hline
\end{tabular}

The reflection, transmission and absorption coefficients of the absorbers are computed in the frequency range 0.01-4 THz using Equations (29), (30), (32) and (33) combined with Equation (11). 
Each laminate $L_{i}$ is modeled through the $\mathrm{ESL}_{i}$-short-line transfer matrix of Equation (26) in which $\sigma_{\mathrm{e} i}(\omega)$ is given by Equation (28) where $N_{i} \sigma_{i}$ is the sheet conductance $G_{s i}$ of the $i$ th laminate. The scattering time $\tau$ and the spacer thickness $t_{\mathrm{sp}}$ are assumed equal to $0.1 \mathrm{ps}$ and $0.035 \mathrm{~mm}$, respectively. The computed frequency spectra of $R, T, A$ and $R_{d B}$ are represented in Figure 5 for different values of $T^{*}$.

It is worth noticing that the absorption coefficient $A$ increases slowly as $T^{*}$ decreases and shows a peak value at $1.1 \mathrm{THz}$, i.e., the first resonant frequency, pretty close to $1.24 \mathrm{THz}$ given by Equation (45) considering $\varepsilon_{\mathrm{r}}=3$. Furthermore, the transmission coefficient $T$ at the resonant frequency assumes values close to the target values $T^{*}$, i.e., $0.1,0.07$ and 0.05 .

The previous calculations are carried out considering the laminate $L_{2}$ as backside reflector. If the backside reflector is made of a PEC layer, i.e., if $T^{*}=0$ and $R_{s 2}=0$, it yields $R_{s 1}=125 \Omega / \mathrm{sq}$. The computed frequency spectra of coefficients $R, T, A$ and of $R_{d B}$ are reported in Figure 6a,b. It is noticed that they are very similar to the ones shown in Figure 5 except for the increased peak value at second resonant frequency.

For the sake of completeness, the input admittance $Y_{\text {in }}$ of the multilayer screen is computed by using Equation (31) with $\tau=0.1 \mathrm{ps}$ and $t_{s p}=0.035 \mathrm{~mm}$. The obtained frequency spectra of the real and imaginary parts of $Y_{\text {in }}$ are shown in Figure $7 \mathrm{a}, \mathrm{b}$ for different values of $T^{*}$.

At a first resonance frequency of $1.1 \mathrm{THz}$ the real and imaginary parts of $Y_{\text {in }}$ are equal to $\left(1 / \eta_{0}\right)=2.65 \mathrm{~ms}$ and zero, respectively, according to the optimal absorbance conditions assumed to obtain the analytical expressions of the sheet conductances.

It is interesting to compare the reflection coefficient computed when a PEC is considered as a backside reflector with the absorbance performance of the adaptive absorber described in [18]. This absorbing screen has a total thickness of $70 \mu \mathrm{m}$, it is backed by a PEC plate and it is made firstly of a graphene lossy sheet and of a multilayer graphene-silicon dioxide $\left(\mathrm{SiO}_{2}\right)$ laminate, sandwiched between two PET dielectric layers. A reflection coefficient of $-18 \mathrm{~dB}$ was obtained at about $1.1 \mathrm{THz}$ when the graphene- $\mathrm{SiO}_{2}$ laminate was electrically doped by a $0.02 \mathrm{~V} / \mathrm{nm}$ electrostatic field bias, giving rise to a laminate-sheet resistance of about $123 \Omega / \mathrm{sq}$ [18]. This value is very close to the one resulting from Equation (44) when the laminate $L_{2}$ is replaced by a PEC layer.

The design of a feasible absorber requires the choice of doped graphene layers characterized by suitable values of sheet resistances in order to obtain laminates having effective sheet resistances close to the ones reported in Table 1 . With this purpose, $\mathrm{HNO}_{3}$ chemically doped graphene layers, characterized by a dc conductivity of $8.33 \mathrm{~ms}$ and a sheet resistance of $120 \Omega / \mathrm{sq}$ [24], should be selected. In fact, values close to $R_{s 1}=135 \mathrm{~ms}$ and $R_{s 2}=31 \mathrm{~ms}$ corresponding to $T^{*}=0.07$ in Table 1 can be obtained using a single doped graphene layer in the first laminate $L_{1}$ and four doped graphene layers in the back laminate $L_{2}$.

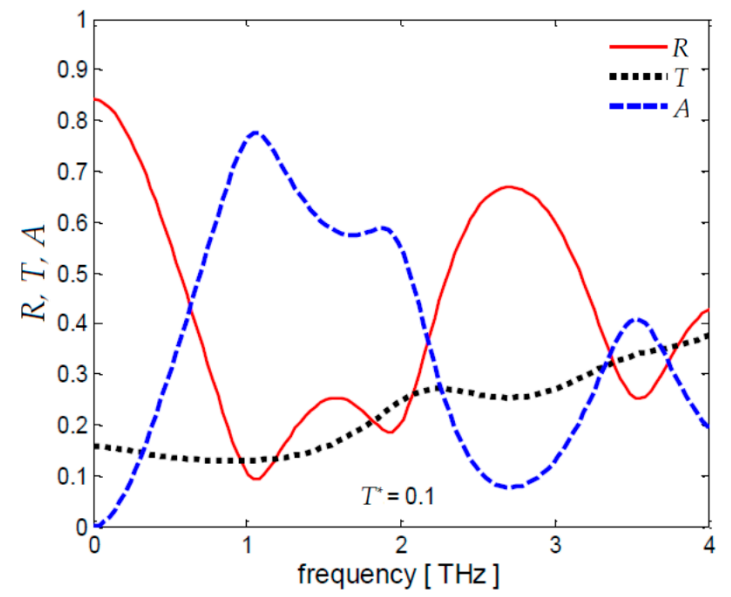

(a)

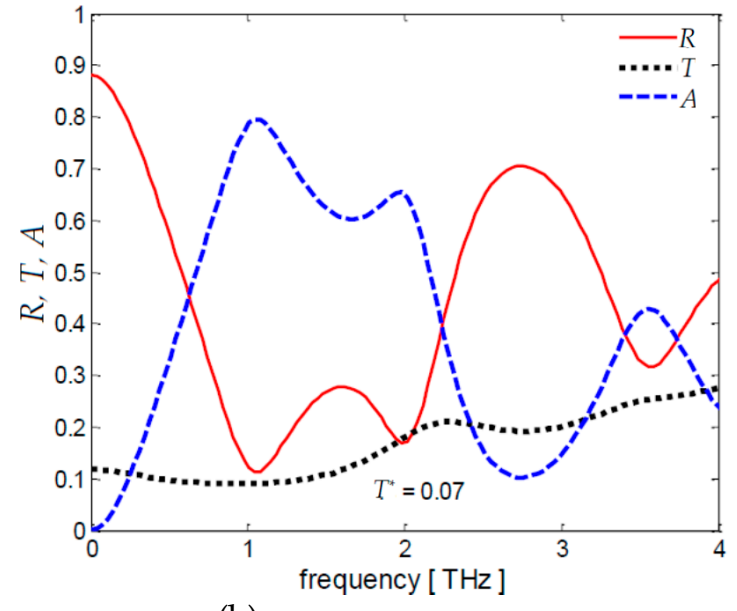

(b)

Figure 5. Cont. 


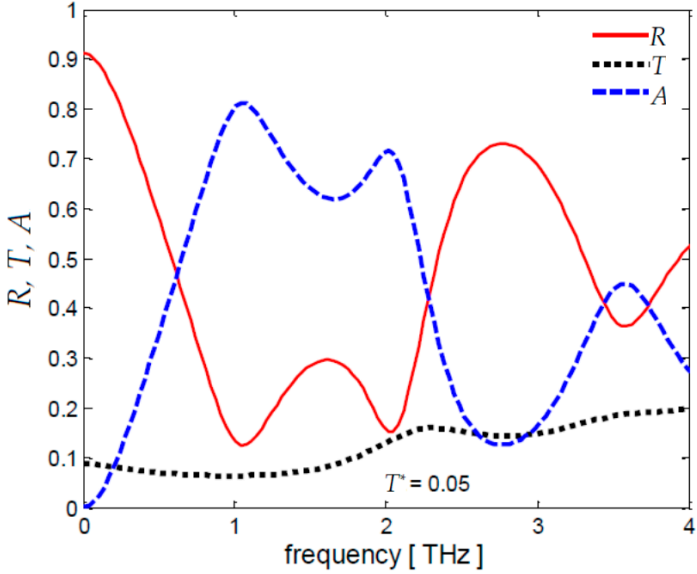

(c)

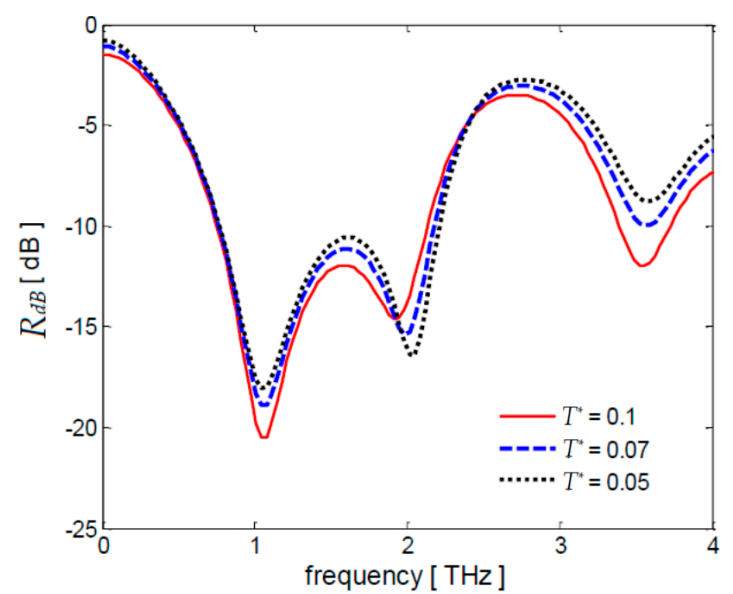

(d)

Figure 5. Frequency spectra of (a-c) coefficients $R, T, A$ and (d) $R_{d B}$ for $\tau=0.1 \mathrm{ps}, t_{s p}=0.035 \mathrm{~mm}$ and different values of $T^{*}$.

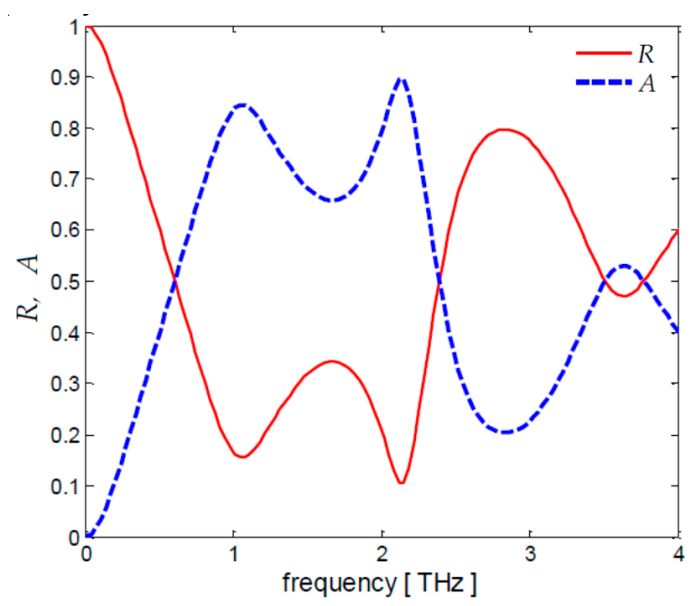

(a)

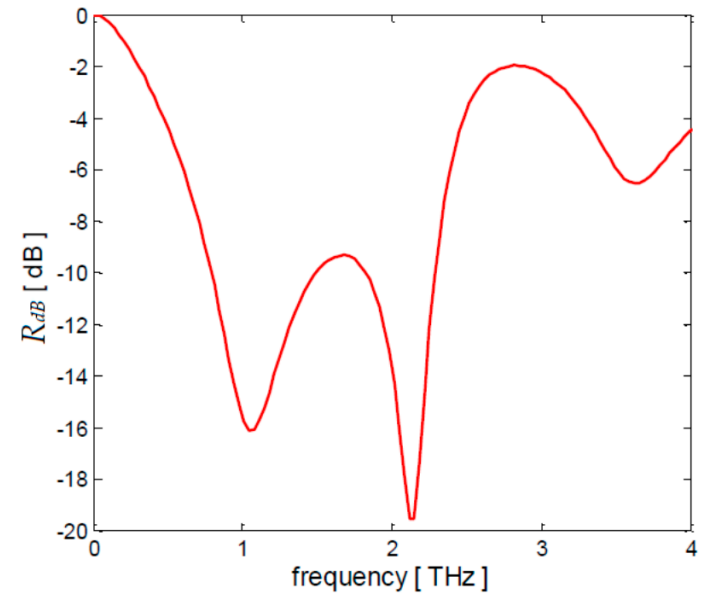

(b)

Figure 6. Frequency spectra of coefficients (a) $R, T, A$ and (b) $R_{d B}$ for $\tau=0.1 \mathrm{ps}, t_{s p}=0.035 \mathrm{~mm}$ in case of a perfect electric conductor (PEC) mirror layer, i.e., $T^{*}=0$.

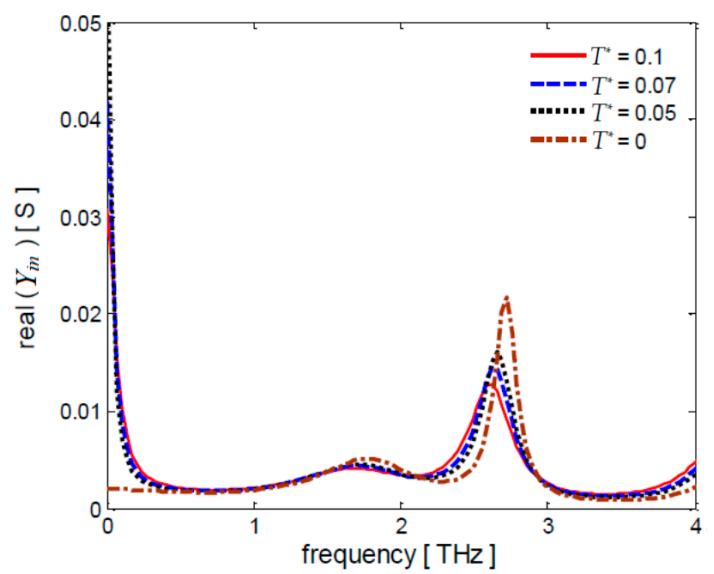

(a)

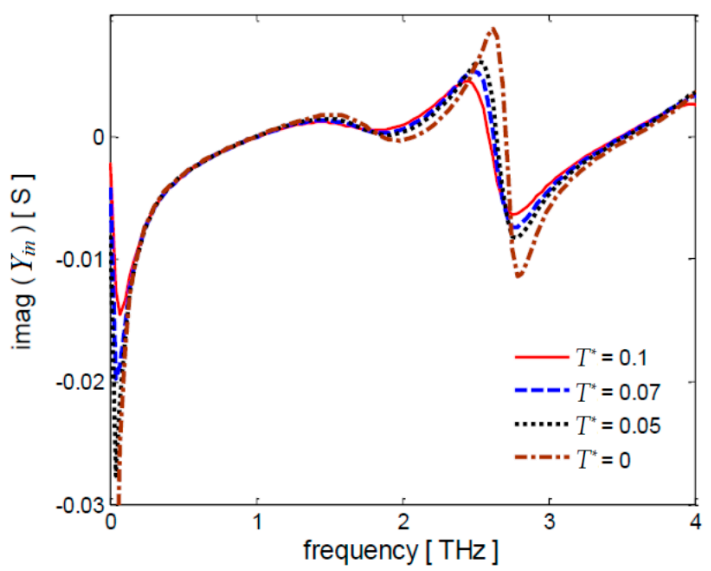

(b)

Figure 7. Frequency spectra of the (a) real and (b) imaginary parts of the input admittance $Y_{\text {in }}$ for $\tau=0.1 \mathrm{ps}, t_{s p}=0.035 \mathrm{~mm}$ and different values of $T^{*}$. 
The coefficients $R, T, A$ and $R_{d B}$ of the designed absorber are computed assuming $\tau=0.1$ ps and $t_{\mathrm{sp}}=0.035 \mathrm{~mm}$, as in the previous applications, and using the overall transfer matrix Equation (11) in which each laminate is represented by the multilayer (ML) matrix of Equation (12) or by the ESL-short-line matrix of Equation (26) obtained considering the sheet resistances of the doped graphene layers and the theoretical ones evaluated in the case $T^{*}=0.07$ (Table 1 ). The obtained results are shown in Figure 8a,b.

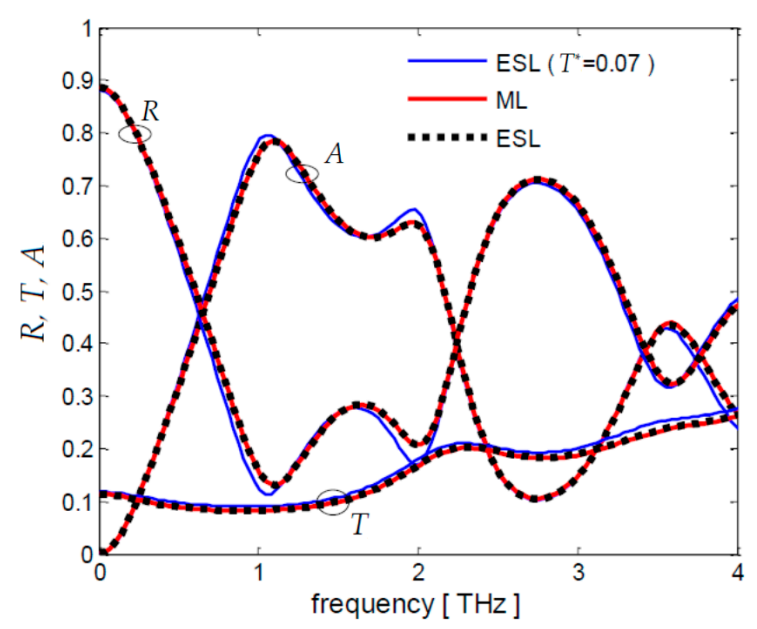

(a)

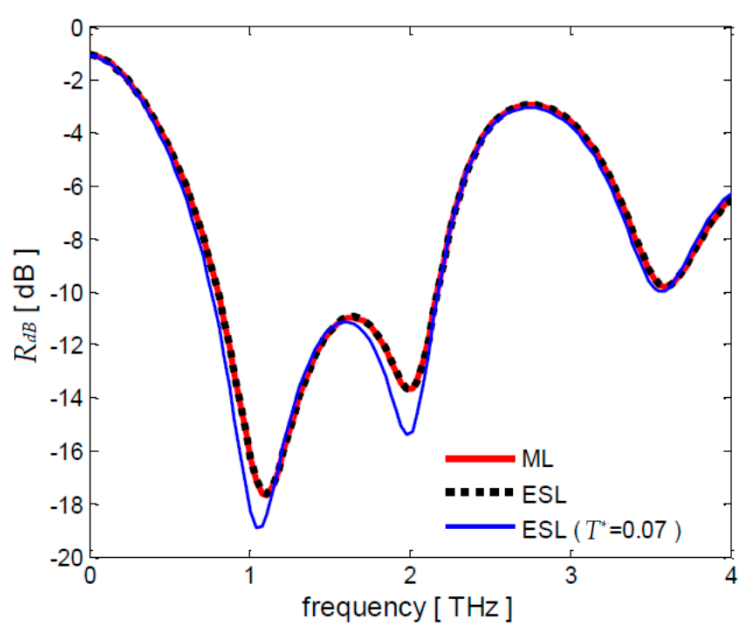

(b)

Figure 8. Frequency spectra of coefficients (a) $R, T, A$ and (b) $R_{d B}$ for the designed screen obtained using ML- and ESL-short-line models. As a comparison, the (a) $R, T, A$ and (b) $R_{d B}$ frequency spectra obtained considering the theoretical sheet resistances obtained for $\mathrm{T}^{*}=0.07$ (Table 1 ) are reported.

It can be noticed that the ML- and ESL-short-line spectra are practically coinciding and they are very close to the ones computed by using the theoretical sheet resistance values. Furthermore, the designed absorber has a $1.4 \mathrm{THz}$ bandwidth centered at $1.5 \mathrm{THz}$ with a reflection coefficient below $-10 \mathrm{~dB}$.

Finally, a sensitivity analysis of the absorption coefficient $A$ is carried out for $T^{*}=0.07$ with respect to different values of $t_{\mathrm{sp}}$ and of $\tau$, setting respectively $\tau=0.1$ ps or $t_{\mathrm{sp}}=0.035 \mathrm{~mm}$. The obtained frequency spectra are represented in Figure $9 a, b$.

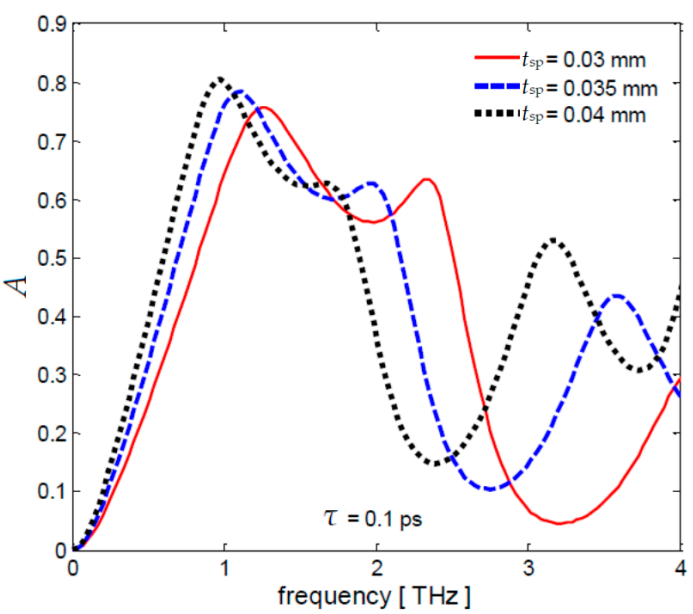

(a)

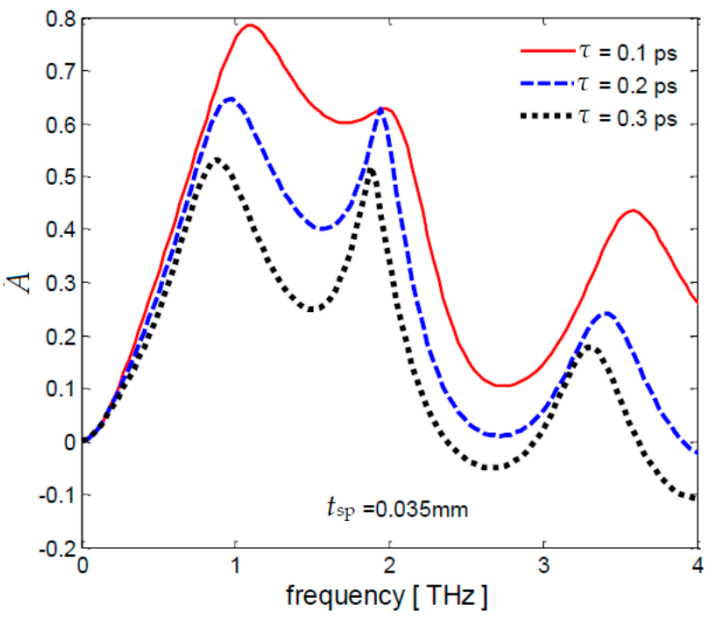

(b)

Figure 9. Frequency spectra of absorber coefficient $A$ for $T^{*}=0.07$ setting (a) $\tau=0.1$ ps and (b) $t_{s p}=0.035 \mathrm{~mm}$. 
The first resonance frequency in Figure $9 \mathrm{a}$ is between $0.9 \mathrm{THz}$ and $1.28 \mathrm{THz}$, depending on the spacer thickness ranging between $0.03 \mathrm{~mm}$ and $0.04 \mathrm{~mm}$. The influence of the scattering time on the frequency spectra is noticeable in Figure $9 b$, showing very different peak values and resonance frequencies for small changes of $\tau$. In fact, the frequency dependence of graphene conductivity is more evident as the scattering time increases. These results highlight the importance of an accurate estimation of the scattering time, that is indicative of the graphene quality since it is strictly related to the carrier mobility and chemical potential [21].

\section{Absorber Transparency}

The optical transmittance $T_{\mathrm{opt}}$ of the absorber structure sketched in Figure 1 is computed by means of Equation (46), considering the back laminate transfer matrix $\left[M_{L 2}\right]$ or the overall matrix $[M]$, respectively given by Equations (48) and (51). In particular, a sensitivity analysis of $T_{\text {opt }}$ with respect to the number of graphene layers $N_{2}$ of the back laminate $L_{2}$ and to the interlayer thickness $t_{\text {int }}$ is carried out. Figure 10a shows $T_{\text {opt }}$ spectra of the back laminate having $t_{\text {int }}=50 \mathrm{~nm}$ and $N_{2}$ ranging between 2 and 5, while Figure $10 \mathrm{~b}$ reports $T_{\text {opt }}$ spectra of the corresponding overall structures, i.e., also considering the matching layer, the laminate $L_{1}$ and the spacer. On the other hand, Figure 10c reports $T_{\text {opt }}$ spectra of the back laminate consisting of five doped graphene layers $\left(N_{2}=5\right)$ and interlayer thickness $t_{\text {int }}$ varying from $50 \mathrm{~nm}$ to $200 \mathrm{~nm}$. Similarly, Figure $10 \mathrm{~d}$ shows $T_{\mathrm{opt}}$ of the corresponding overall structures, i.e., also considering the matching layer, the laminate $L_{1}$ and the spacer.

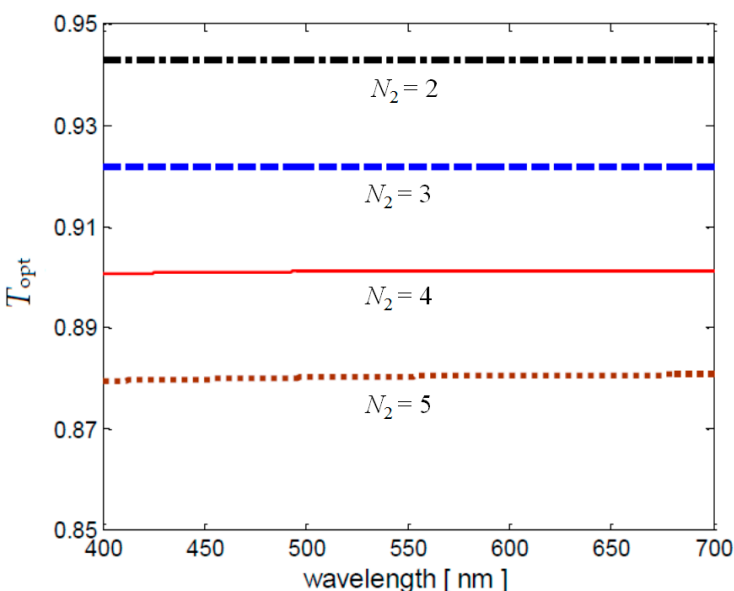

(a)

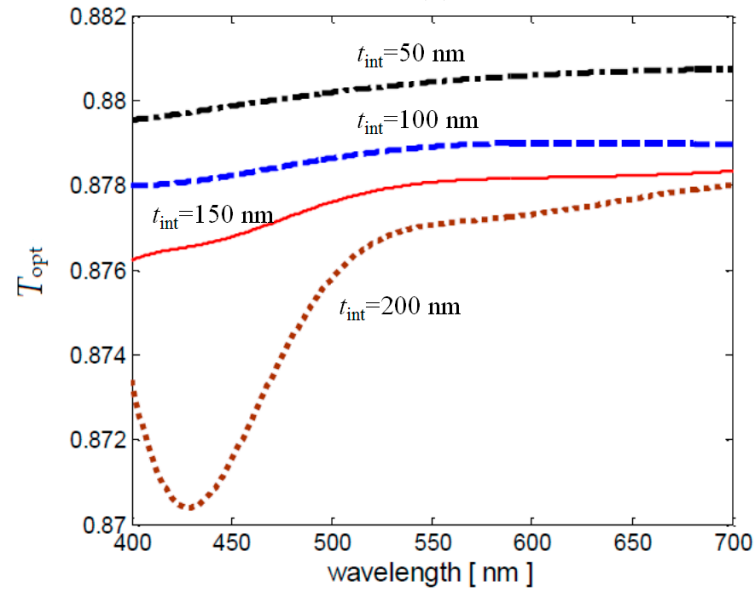

(c)

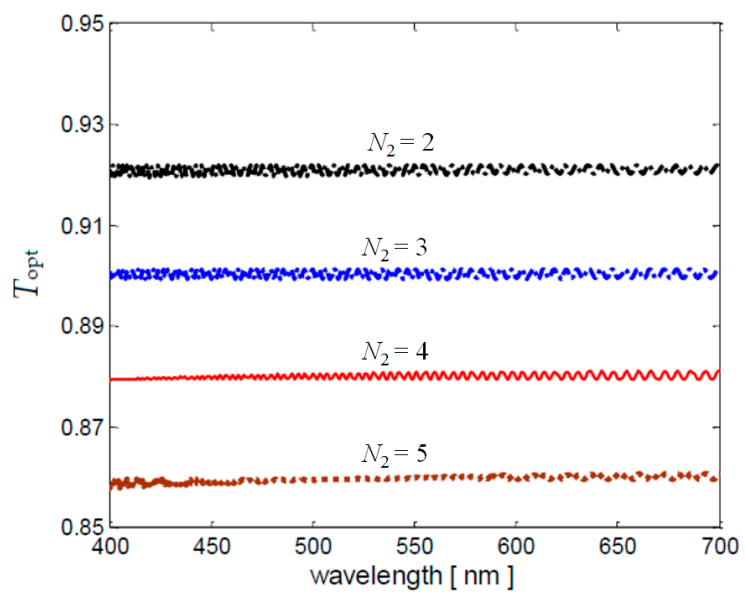

(b)

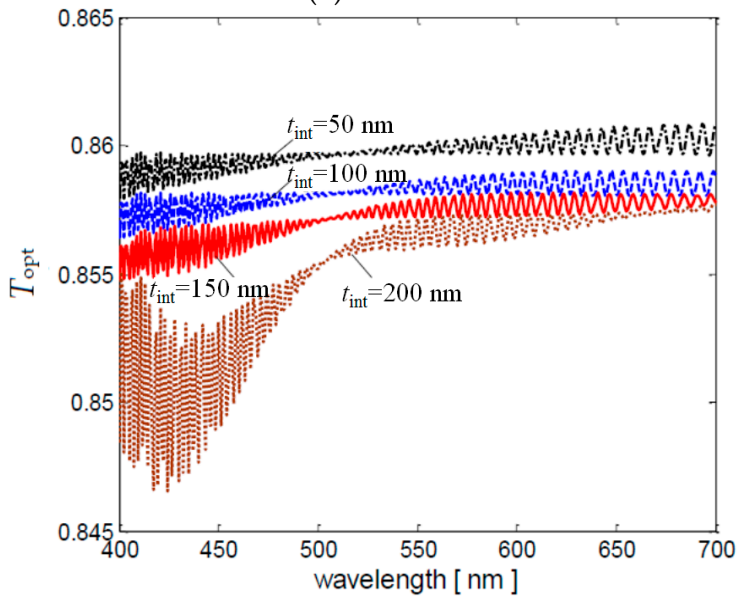

(d)

Figure 10. Optical transmittance $T_{\text {opt }}$ of the $(\mathbf{a}, \mathbf{c})$ back laminate $L_{2}$ and $(\mathbf{b}, \mathbf{d})$ of the overall absorbing structure. The spectra are computed in the visible range considering $t_{\text {int }}=50 \mathrm{~mm}$ and $N_{2}$ ranges between 2 and $5(\mathbf{a}, \mathbf{b})$ or $N_{2}=5$ and $t_{\text {int }}$ varying from $50 \mathrm{~nm}$ to $200 \mathrm{~nm}(\mathbf{c}, \mathbf{d})$. 
It can be noticed that $T_{\text {opt }}$ is always greater than $85 \%$. Moreover, when the PET interlayer thickness is one order of magnitude lower than the considered wavelength, $T_{\text {opt }}$ can be easily computed by means of the approximated expression Equation (8). As the PET interlayer thickness increases, $T_{\text {opt }}$ cannot be computed by means of Equation (8), as shown in Figure 10c. In fact, mutual reflections inside the interlayer are no more than negligible.

Finally, from Figure $10 \mathrm{~b}$ it results that $T_{\mathrm{opt}}$ of the designed screen (with $N_{2}=4$ ) is around $88 \%$ in all the visible ranges.

\section{Conclusions}

The proposed absorber structure consists of a front matching PET layer followed by a graphene/PET laminate, a PET spacer and a back graphene/PET laminate. The matching layer and the spacer have one-quarter-wavelength thickness. The structure is extremely simple since it does not include complex frequency-selective surfaces or metamaterials. Moreover, the designed absorbers have a total thickness of $70 \mu \mathrm{m}$ and they are transparent and metal free, since no metal is used, also for the backside reflector.

The absorption performances are evaluated through numerical calculations in the low-terahertz frequency range and both graphene conductivity and PET permittivity are considered as frequency-dependent quantities. A homogenization procedure is applied to represent the periodic graphene-PET structures as equivalent short-line single layers.

The optimum sheet resistances of the laminates are obtained analytically as functions of the interlayer relative permittivity and of the target transmission coefficient at the first resonance frequency. It is worth noticing that the analytical expressions of the optimum laminate-sheet resistances can be applied to both low-gigahertz or low-terahertz frequency ranges. Then, $\mathrm{HNO}_{3}$ chemically doped graphene layers are considered in order to design feasible absorbing screens with laminate-sheet resistances comparable with the theoretical ones. The computed frequency spectra of the absorption, reflection and transmission coefficients in the frequency range up to $4 \mathrm{THz}$ prove the good performance of the designed absorbers and the utility of the proposed design method. In particular, the proposed screen has a $1.4 \mathrm{THz}$ bandwidth centered at $1.5 \mathrm{THz}$ with reflection coefficients below - $10 \mathrm{~dB}$ and an absorption coefficient with peak values around 0.8 at the first resonant frequency of $1.1 \mathrm{THz}$. Then, a sensitivity analysis is carried out to highlight the strong influence of the estimated scattering time on the screen performances.

Finally, the optical transmittance of the proposed absorber is computed by means of the optical matrix theory and we conclude that such transmittance is greater than $88 \%$ in all the visible ranges.

Author Contributions: Conceptualization, methodology, validation, formal analysis, investigation, writing, review and editing, A.G.D., M.D., M.S.S.; software, A.G.D. All authors have read and agreed to the published version of the manuscript.

Funding: This research received no external funding.

Acknowledgments: The authors thank Francesca Sarto (ENEA) for technical discussions about optical transmittance calculations.

Conflicts of Interest: The authors declare no conflict of interest.

\section{References}

1. Nagatsuma, T.; Ducournau, G.; Renaud, C.C. Advances in terahertz communications accelerated by photonics. Nat. Photonics 2016, 10, 371. [CrossRef]

2. Song, H.J.; Nagatsuma, T. Present and future of terahertz communications. IEEE Trans. Terahertz Sci. Technol. 2011, 1, 256-263. [CrossRef]

3. Chen, M.; Sun, W.; Cai, J.; Chang, L.; Xiao, X. Frequency-tunable terahertz absorbers based on graphene metasurface. Opt. Commun. 2017, 382, 144-150. [CrossRef]

4. Wang, Y.; Song, M.; Pu, M.; Gu, Y.; Hu, C.; Zhao, Z.; Wang, C.; Yu, H.; Luo, X. Staked graphene for tunable terahertz absorber with customized bandwidth. Plasmonics 2016, 11, 1201-1206. [CrossRef] 
5. Landy, N.I.; Sajuyigbe, S.; Mock, J.J.; Smith, D.R.; Padilla, W.J. Perfect metamaterial absorber. Phys. Rev. Lett. 2008, 100, 207402. [CrossRef]

6. Watts, C.M.; Liu, X.; Padilla, W.J. Metamaterial electromagnetic wave absorbers. Adv. Mater. 2012, 24, OP98-OP120. [CrossRef]

7. Min Woo, J.; Kim, M.S.; Woong Kim, H.; Jang, J.H. Graphene based salisbury screen for terahertz absorber. Appl. Phys. Lett. 2014, 104, 081106. [CrossRef]

8. Baringhaus, J.; Ruan, M.; Edler, F.; Tejeda, A.; Sicot, M.; Taleb-Ibrahimi, A.; Li, A.-P.; Jiang, Z.; Conrad, E.H.; Tegenkamp, C.; et al. Exceptional ballistic transport in epitaxial graphene nanoribbons. Nature 2014, 506, 349-354. [CrossRef]

9. Faraji, M.; Moravvej-Farshi, M.K.; Yousefi, L. Tunable THz perfect absorber using graphene-based metamaterials. Opt. Commun. 2015, 355, 352-355. [CrossRef]

10. Fardoost, A.; Vanani, F.G.; Amirhosseini, A.A.; Safian, R. Design of a multilayer graphene-based ultrawideband terahertz absorber. IEEE Trans. Nanotechnol. 2016, 16, 68-74.

11. Zhang, Y.; Feng, Y.; Zhu, B.; Zhao, J.; Jiang, T. Graphene based tunable metamaterial absorber and polarization modulation in terahertz frequency. Opt. Express 2014, 22, 22743-22752. [CrossRef] [PubMed]

12. Chang, Y.C.; Liu, C.H.; Liu, C.H.; Zhang, S.; Marder, S.R.; Narimanov, E.E.; Zhong, Z.; Norris, T.B. Realization of mid-infrared graphene hyperbolic metamaterials. Nat. Commun. 2016, 7, 1-7. [CrossRef] [PubMed]

13. Daraei, O.M.; Goudarzi, K.; Bemani, M. A tunable ultra-broadband terahertz absorber based on two layers of graphene ribbons. Opt. Laser Technol. 2020, 122, 105853. [CrossRef]

14. Soleymani, A.; Meymand, R.E.; Granpayeh, N. Broadband near-perfect terahertz absorber in single-layered and non-structured graphene loaded with dielectrics. Appl. Opt. 2020, 59, 2839-2848. [CrossRef]

15. Aladadi, Y.T.; Alkanhal, M.A.S. Extraction of the terahertz constitutive tensors of multilayer graphene-dielectric stacks. Opt. Commun. 2020, 464, 125487. [CrossRef]

16. Xing, R.; Dong, C.; Zhu, B.; Gao, Y.; Jian, S. Numerical analysis on ribbon-array-sheet coupled graphene terahertz absorber. IEEE Photonics Technol. Lett. 2016, 28, 2207-2210. [CrossRef]

17. Dong, Y.; Liu, P.; Yu, D.; Li, G.; Yang, L. A tunable ultrabroadband ultrathin terahertz absorber using graphene stacks. IEEE Antennas Wirel. Propag. Lett. 2016, 16, 1115-1118. [CrossRef]

18. D'Aloia, A.G.; D'Amore, M.; Sarto, M.S. Adaptive terahertz absorber based on tunable graphene multilayer. In Proceedings of the 2017 International Symposium on Electromagnetic Compatibility-EMC EUROPE, Angers, France, 4-7 September 2017; pp. 1-6.

19. D'Aloia, A.G.; D'Amore, M.; Sarto, M.S. Terahertz Shielding Prediction of Id-Periodic N Anolayered Coatings by an Effective Homogeneous Model. In Proceedings of the 2018 International Symposium on Electromagnetic Compatibility (EMC EUROPE), Amsterdam, The Netherlands, 27-30 August 2018; pp. 772-777.

20. D'Amore, M.; D'Aloia, A.G.; Sarto, M.S. Terahertz shielding effectiveness and optical transmittance of graphene multilayer thin sheets. In Proceedings of the 2014 International Symposium on Electromagnetic Compatibility, Gothenburg, Sweden, 1-4 September 2014; pp. 482-487.

21. D'Aloia, A.G.; D'Amore, M.; Sarto, M.S. Terahertz shielding effectiveness of graphene-based multilayer screens controlled by electric field bias in a reverberating environment. IEEE Trans. Terahertz Sci. Technol. 2015, 5, 628-636. [CrossRef]

22. D'Aloia, A.G.; D'Amore, M.; Sarto, M.S. Optimal terahertz shielding performances of flexible multilayer screens based on chemically doped graphene on polymer substrate. In Proceedings of the 2015 IEEE International Symposium on Electromagnetic Compatibility (EMC), Gothenburg, Sweden, 1-4 September 2014; pp. 1030-1035.

23. Macleod, H.A. Thin-Film Optical Filters; CRC press: Boca Raton, FL, USA, 2010.

24. Bae, S.; Kim, H.; Lee, Y.; Xu, X.; Park, J.S.; Zheng, Y.; Lei, T.; Kim, H.R.; Kim, K.S.; Kim, Y.J.; et al. Roll-to-roll production of 30-inch graphene films for transparent electrodes. Nat. Nanotechnol. 2010, 5, 574. [CrossRef]

25. Hanson, G.W. Dyadic Green's functions for an anisotropic, non-local model of biased graphene. IEEE Trans. Antennas Propag. 2008, 56, 747-757. [CrossRef]

26. Ju, L.; Geng, B.; Horng, J.; Girit, C.; Martin, M.; Hao, Z.; Bechtel, H.A.; Liang, X.; Zettle, A.; Wang, F.; et al. Graphene plasmonics for tunable terahertz metamaterials. Nat. Nanotechnol. 2011, 6, 630. [CrossRef]

27. Jablan, M.; Buljan, H.; Soljacic, M. Plasmonics in graphene at infrared frequencies. Phys. Rev. B 2009, 80, 245435. [CrossRef] 
28. Havriliak, S.; Negami, S. A complex plane representation of dielectric and mechanical relaxation processes in some polymers. Polymer 1967, 8, 161-210. [CrossRef]

29. Fedulova, E.V.; Nazarov, M.M.; Angeluts, A.A.; Kitai, M.S.; Sokolov, V.I.; Shkurinov, A.P. Studying of dielectric properties of polymers in the terahertz frequency range. In Saratov Fall Meeting 2011: Optical Technologies in Biophysics and Medicine XIII.; International Society for Optics and Photonics: Saratov, Russia, 2012; p. 83370I.

30. Kang, M.H.; Milne, W.I.; Cole, M.T. Doping stability and opto-electronic performance of chemical vapour deposited graphene on transparent flexible substrates. IET Circuits Devices Syst. 2015, 9, 39-45. [CrossRef]

31. Dawlaty, J.M.; Shivaraman, S.; Strait, J.; George, P.; Chandrashekhar, M.; Rana, F.; Spencer, M.G.; Veksler, D.; Chen, Y. Measurement of the optical absorption spectra of epitaxial graphene from terahertz to visible. Appl. Phys. Lett. 2008, 93, 131905. [CrossRef]

32. Nair, R.R.; Blake, P.; Grigorenko, A.N.; Novoselov, K.S.; Booth, T.J.; Stauber, T.; Peres, N.M.R.; Geim, A.K. Fine structure constant defines visual transparency of graphene. Science 2008, 320, 1308. [CrossRef]

33. Blake, P.; Hill, E.; Castro Neto, A.H.; Novoselov, K.S.; Jiang, D.; Yang, R.; Booth, T.J.; Geim, A.K. Making graphene visible. Appl. Phys. Lett. 2007, 91, 063124. [CrossRef]

34. Bruna, M.; Borini, S. Optical constants of graphene layers in the visible range. Appl. Phys. Lett. 2009, 94, 031901. [CrossRef]

35. Elman, J.F.; Greener, J.; Herzinger, C.M.; Johs, B. Characterization of biaxially-stretched plastic films by generalized ellipsometry. Thin Solid Films 1998, 313, 814-818. [CrossRef]

36. D'Aloia, A.G.; D'Amore, M.; Sarto, M.S. Effective medium model of periodic nanolayered transparent shields. IEEE Trans. Electromagn. Compat. 2019, 61, 1803-1810. [CrossRef]

37. D'Aloia, A.G.; D'Amore, M.; Sarto, M.S. Transparent Graphene-Based Absorber for Next Generation Wireless 5G Technology. In Proceedings of the 2019 International Symposium on Electromagnetic Compatibility-EMC EUROPE, Barcelona, Spain, 2-6 September 2019; pp. 360-365.

38. Dobrowolski, J.A. Optical properties of films and coatings. Handb. Opt. I 1995, 42, 3-130.

(C) 2020 by the authors. Licensee MDPI, Basel, Switzerland. This article is an open access article distributed under the terms and conditions of the Creative Commons Attribution (CC BY) license (http://creativecommons.org/licenses/by/4.0/). 